\title{
Effect of Extracellular Vesicles on Neural Functional Recovery and Immunologic Suppression after Rat Cerebral Apoplexy
}

\author{
Beilei Hua Songfang Chen ${ }^{a}$ Ming Zou ${ }^{a}$ Zhiyong Hea Shengmin Shao ${ }^{a}$ \\ Baohua Liub \\ a Department of Neurology, ${ }^{b}$ Department of Rehabilitation Medicine, The $2^{\text {nd }}$ Affiliated Hospital of \\ Wenzhou Medical University, Wenzhou, China
}

\section{Key Words}

Cerebral apoplexy - Mesenchymal stem cells • Neural functional recovery • Immunologic suppression

\begin{abstract}
Background: Previous studies have demonstrated that mesenchymal stem cells (MSCs) can promote the recovery of neural function after cerebral apoplexy by secreting multiple cytokines. In addition, cell factor-derived extracellular vesicles play an important role in recovery of neural function. The aim of this study was to determine the effect of extracellular vesicles on neural functional recovery and brain tissue remodeling after cerebral apoplexy in a rat model. Methods: The rat models with local ischemic stroke was established and three random groups were created. In groups A and B, human bone marrow-derived MSCs and MSC-derived extracellular vesicles were transplanted into rats. In the control group (group C), only normal saline was injected. Then, we evaluated motor coordination ability, pathologic changes of the brain, immune responses in the central and peripheral nervous systems, regeneration of blood vessels, and nervous tissue in 4 weeks after cerebral apoplexy. Results: Obvious regeneration of blood vessels and nervous tissues were identified in groups $A$ and $B$. There was no significant difference with respect to coordination between groups $A$ and $B$, but coordination in groups $A$ and $B$ was significantly better than the control group. Immunohistochemical staining of brain tissue showed that extracellular vesicles exerted no effect on infiltration of immune cells in the central nervous system. Weakened immune suppression was noted 1 week after cerebral apoplexy, which provided a favorable environment for remodeling of brain tissue. Conclusion: MSC-derived extracellular vesicles accelerated neural functional recovery after cerebral apoplexy. The weakened immune suppression was beneficial to remodeling of brain tissue.
\end{abstract}




\section{Introduction}

Previous research has demonstrated that stem cell therapy can induce functional reconstruction of brain tissues and reduce brain tissue injury, and is thus beneficial to recovery of neurologic function [1-7]. Such a therapeutic effect is not limited to a single stem cell line; relevant functions can also be found in other types of precursor cells. Mesenchymal stem cells (MSCs) are highly favored because of their extensive sources [1]. The initial aim of stem cell therapy is to replace lost brain tissue, but the latest evidence maintains that MSCs can always prevent the secondary degeneration of neurons, regulate the central and peripheral immune responses, and promote the vascular nerve regeneration in brain tissue. Previously, models of myocardial ischemia have been established. Treatment of hepatic fibrosis, kidney injury, and cerebral ischemia has confirmed that MSCs are effective when administered via small extracellular vesicles. The exosome is the main form of outer vesicles, and probably plays an important role in cell-cell communication by combining with plasma membranes to release encoded and non-encoded DNA, lipid, and protein. Recent reports have seldom found severe complications after treatment of cerebral ischemia by MSCs, but we cannot exclude the possibility of MSCs inducing small vessel occlusion. In addition, no evidence has shown an increased risk of tumors following stem cell treatment, but considering the possibility of tumor formation induced by embryonic and other types of stem cells, the related risk of MSCs cannot be completely excluded [2,3]. In contrast, the advantages of extracellular vesicle treatment might be more obvious, which is not only consistent with the indirect action of stem cells, but also avoids the incidence of tumors after stem cell therapy. Therefore, we chose to use MSCs and extracellular vesicles transplanted in a rat model. The aim of this study was to evaluate the effect of extracellular vesicles on neural functional recovery and brain tissue remodeling in a rat stroke model, which could provide references and guidance for basic research and clinical treatment.

\section{Materials and Methods}

\section{Animal grouping}

Forty-five 10-week old rats were purchased from Beijing Weitong Lihua Experimental Animal Technology Co. Ltd. All of the rats were randomly divided into groups A (extracellular vesicle), B (MSC), or C (blank control group), with 15 rats per group. All of the rats were raised under standard conditions with free access to food and water. All of the rats were treated by middle cerebral artery occlusion (MCAO) according to the method reported in the literature [8]. A silica gel-coated single fiber wire (Doccol Corp) was introduced into the left common carotid artery (CCA) of rats, then carefully advanced into the left middle cerebral artery (MCA). A probe was used to record laser Doppler blood flow (LDF) during the procedure. Thirty min later, the single fiber yarn was removed and reperfusion of blood vessels was achieved. LDF was repeatedly recorded for $15 \mathrm{~min}$ before closing the wound. MSCs were administered at an intravenous infusion of $1 \times 10^{4}$ cells in $0.5 \mathrm{ml}$ of normal saline, and an equivalent amount of normal saline was delivered for the rats in groups B and C. MSC-derived extracellular vesicles were diluted at a density of $2.46 \times 10^{4}$ extracellular vesicles with $0.5 \mathrm{ml}$ of normal saline. The rats were anesthetized after $24 \mathrm{~h}$ and stem cell transplantation was performed through a right femoral venous cannula. The rats were e-injected on the $3^{\text {rd }}$ and $5^{\text {th }}$ days after ischemia; the average infusion rate of the injection was $0.5 \mathrm{ml}$ per $5 \mathrm{~min}$. All of the procedures performed in this study were approved by the Ethics Committee of our hospital.

Culture of MSCs and extracellular vesicles

Bone marrow aspirates were obtained from the posterior iliac crest of study subjects following informed consent. Bone marrow derived-MSCs were cultured in 6-well plates after separation and gradient centrifugation, then supplemented with $5 \%$ platelet lysis, $1 \%$ glutamine, and $1 \%$ penicillin-streptomycin. Non-adherent cells were removed after $24 \mathrm{~h}$, and the immunophenotype of culture-expanded MSCs was characterized by flow cytometry. MSCs were then incubated by fluorescein isothiocyanate combined with phycoerythrin or anti-CD90, anti-CD105, anti-CD14, anti-CD31, anti-CD73, anti-CD146, anti-, or anti-CD45 antibody of allophycocyanin at $4^{\circ}$ for $20 \mathrm{~min}$, then the dead cells were removed using propidium iodide. 


\section{Cellular Physiology Cell Physiol Biochem 2016;40:155-162 \begin{tabular}{l|l|l}
\hline DOI: 10.1159/000452533 & $\begin{array}{l}\text { (c) 2016 The Author(s). Published by S. Karger AG, Basel } \\
\text { www.karger.com/cpb }\end{array}$
\end{tabular} \\ Hu et al.: Extracellular Vesicles Facilitate Regeneration}

A matched control monoclonal antibody was applied to confirm the level of background staining in all observations, and FC500 flow cytometry was used.

After 3 cell passages, the MSC culture media was collected every $48 \mathrm{~h}$. Vacuoles and fragments were removed over $0.22-\mathrm{mm}$ filtration pores. The culture media was placed at a temperature of $-20^{\circ} \mathrm{C}$. The culture media was mixed with $3 \mu / \mathrm{ml}$ of heparin and $3 \mathrm{mg} / \mathrm{ml}$ of Actilyse, and the mixture was incubated at a temperature of $37^{\circ} \mathrm{C}$ for $3 \mathrm{~h}$. According to previous reports, extracellular vesicles were concentrated using the polyethylene glycol (PEG) precipitation method [9]. The extracellular vesicle samples were quantitatively measured using a polymerase chain reaction (PCR) and serologic infection. The level of extracellular vesicle protein expression was measured using a micro-Quinaldic acid and immunoblotting assay. The samples were transferred to a polyvinyl fluoride film, and the membranes were stained with Tsg101 or CD81 antibody against the external marker protein.

\section{Behavioral testing}

Prior to establishment of the rat model with cerebral apoplexy, the rats ( $\mathrm{n}=10$ rats for each group) were tested for 4 min by two blinded examiners. The motor performance was tested using the BassoBeattie-Bresnahan (BBB) 21-point open field locomotor scale. BBB scores, divide combinations of rat hindlimb movement, trunk position and stability, stepping, coordination, paw placement, toe clearance, and tail position, where 0 represents no locomotion and 21 indicates normal motor function.

\section{Immunohistochemical staining}

First, five rats from each group were anaesthetized with $7 \%$ chloral hydrate $(200 \mathrm{mg} / \mathrm{kg})$, then the brain tissues were obtained after infusing phosphate buffer solution (PBS) through the heart. The infarct size was quantitatively calculated according to the following equation: the total area of the ipsilateral hemisphere was subtracted from the area of non-injured contralateral hemisphere. The lesion volume is presented as a volume percentage of the lesion compared with the contralateral hemisphere (Fig. 1A). The sections were stained with mouse monoclonal anti-NeuN antibody (1:1000 dilution), and goat anti-mouse Alexa Fluor 488 antibody was used for evaluating the survival of neurons ( 2 sections for each rat). Leukocyte infiltration was detected by rat anti-mouse CD45 antibody (1:100 dilution) and donkey anti-rat Alexa antibody Fluor 488 antibody, and the same region was chosen for evaluating the CD45+ white cell density.

Five-bromine-2'-deoxyuridine was injected into the abdominal cavity 8-18 days after cerebral apoplexy, then endogenous cell proliferation and differentiation of newborn cells were assessed. The sections were immunohistochemically-stained with BrdU, microtubule-associated protein, NeuN, and CD31 (clone 10G9; 2:100 dilution). Antigen retrieval was performed for CD31. The antibodies included monoclonal mouse antiBrdU (1:400), monoclonal anti-BrdU antibody (1:400), and multi-clone goat anti-DCX antibody (1:50). All of the antibodies were purchased from BD Biosciences Company (BD Biosciences, CA, USA).

\section{Flow cytometry}

The single cell suspension in previous experiments was used. The absolute number of white blood cells was counted using a blood analyzer. In addition, red blood cells were separated. The cerebral hemispheres from 5 rats in each group were resected and centrifuged at $286 \mathrm{~g}$ for $5 \mathrm{~min}$ at $18^{\circ} \mathrm{C}$. The supernatant was abandoned, re-suspended, and centrifuged in $15 \mathrm{ml}$ of HCL/PBS at $2800 \mathrm{~g}$ for $20 \mathrm{~min}$. The treated cells were washed twice with $0.01 \mathrm{M}$ PBS. The isolated cells were incubated with rat anti-mouse antibody CD16/CD32 for $15 \mathrm{~min}$ at $4^{\circ} \mathrm{C}$. The absolute number of cells was calculated by the product of the white blood cell count per microliter and the percentage of individual subsets. The leukocyte infiltration in the brain tissues was quantitatively assessed based on the level of CD45 expression. Leukocyte subsets were further divided into leukomonocytes and marrow cells according to

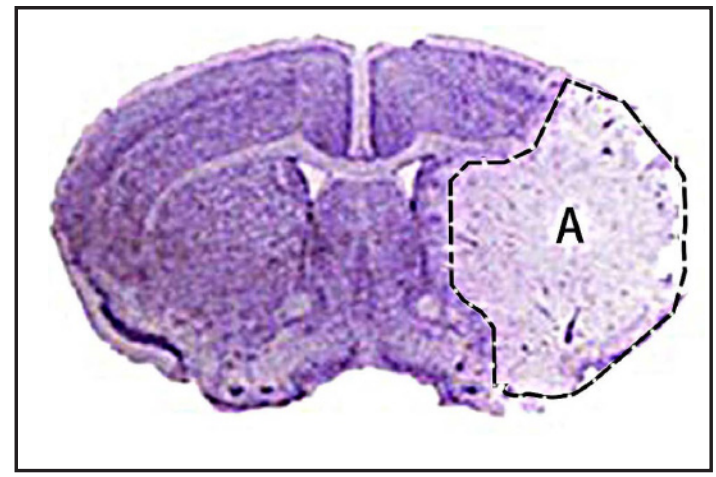

Fig. 1. An area shows the brain sections were stained with cresyl violet in the infarction area after being treated with MCAO. 
the expression of blood cell antigens and the source of brain tissue by multi-channel flow cytometry, which included neutrophil granulocytes, macrophages, hyaline leukocytes, and dendritic cells.

\section{Statistical analysis}

SPSS 17.0 statistical software was used for data analysis (SPSS, Inc., Chicago, IL, USA). Comparisons between the two groups were performed using a two-tailed independent $t$-test. Multi-group comparisons were performed using a post hoc Tukey's test. All data are expressed as the mean \pm standard deviation. A $P$ value $<0.05$ was considered statistically significant.

\section{Results}

Extracellular vesicles and MSCs effectively reduce movement coordination disorder after cerebral ischemia

In the current study we systematically compared the effect of MSCs and extracellular vesicles on promoting recovery of neuromotor function after cerebral apoplexy. On days 1,3 , and 5 after cerebral apoplexy, MSCs and extracellular vesicles were transplanted intravenously in a rat model. Motor coordination in the MSC and extracellular vesicle groups was significantly improved compared with the control group 2 weeks after cerebral ischemia, but no significant difference was noted between the two groups (Fig. 2).

\section{Effect of MSCs and extracellular vesicles on neural function recovery}

We determined survival of brain striatal neurons 4 weeks after cerebral apoplexy, which was consistent with the reduction in neural lesions. The density of NeuN+neure in the MSC and extracellular vesicle groups was higher than the blank control group, but there was no obvious difference between the MSC and extracellular vesicle groups. To ascertain whether or not extracellular vesicles promote nerve regeneration, we compared the changes in cell multiplication for the MSC and extracellular vesicle groups 4 weeks after the establishment of a cerebral apoplexy model. Compared with the control group, BrdU+ cells were mainly found in the striatum of rats treated with MSCs and extracellular vesicles. The number of

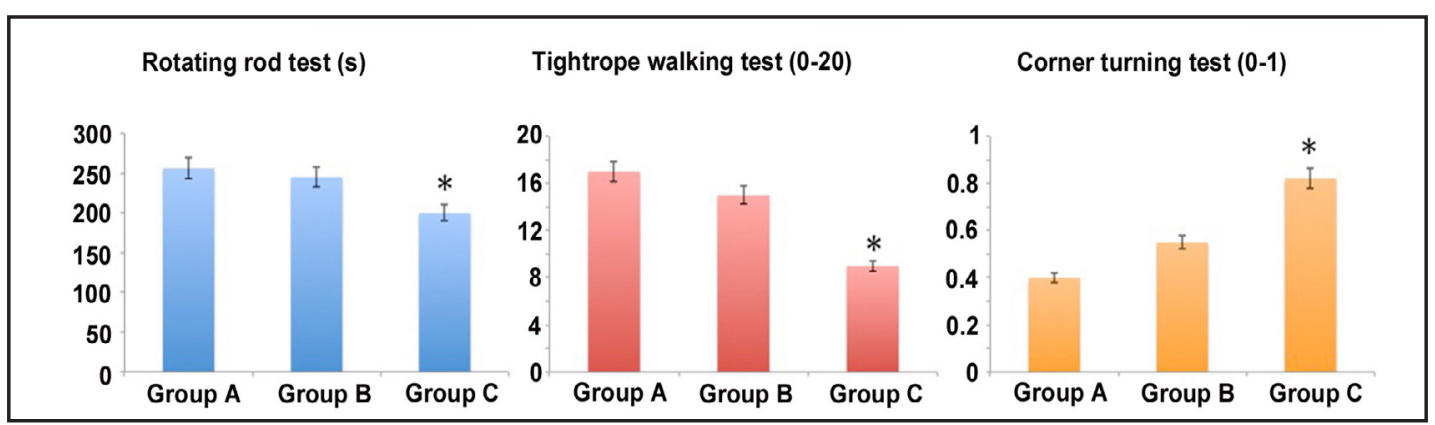

Fig. 2. Evaluation of motor coordination ability for each group 2 weeks after cerebral apoplexy focal cerebral ischemia. Groups A and B were clearly higher than the blank control group, but there was no obvious difference between the two groups, * represents statistical significance compared with groups A and B.

Table 1. Peripheral blood immune cell level of the mice in each group (the number of cells per microliter blood). * represents statistical significance compared with groups $\mathrm{A}$ and $\mathrm{B}$

\begin{tabular}{llll}
\hline & Group A & Group B & Group C \\
\hline B cell & $1084.3 \pm 523.5$ & $998.2 \pm 614.4$ & $1145.4 \pm 477.6$ \\
NK cell & $24.0 \pm 7.4$ & $23.4 \pm 6.2$ & $29.1 \pm 8.7$ \\
T cell & $388.4 \pm 36.3$ & $365.7 \pm 44.0$ & $400.5 \pm 48.3$ \\
Hyaline leukocyte & $122.2 \pm 104.6$ & $188.7 \pm 98.1$ & $577.1 \pm 122.6^{*}$ \\
Dendritic cell & $4.7 \pm 3.2$ & $4.6 \pm 2.9$ & $3.6 \pm 2.1^{*}$ \\
\hline
\end{tabular}




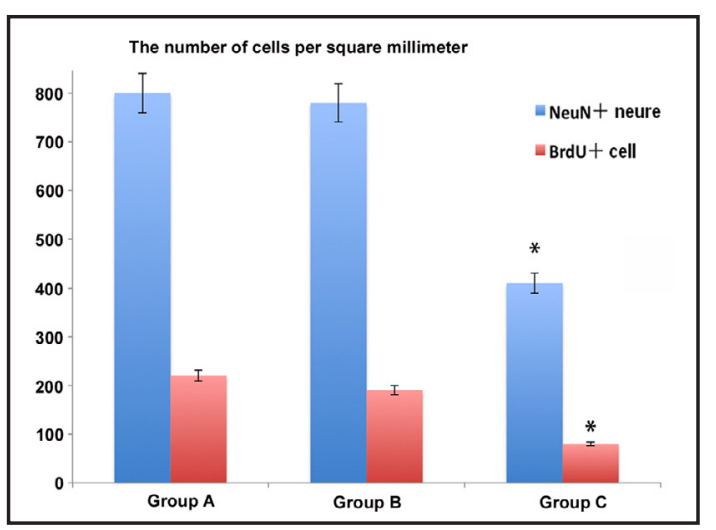

Fig. 3. The density of NeuN + neurons and $\mathrm{Br}$ $\mathrm{dU}$-marked neurons was detected in the brain tissue for each group in the $4^{\text {th }}$ week. There was no significant difference between groups $\mathrm{A}$ and $\mathrm{B}$, which were all higher than group C. * represents statistical significance compared with groups A and B.

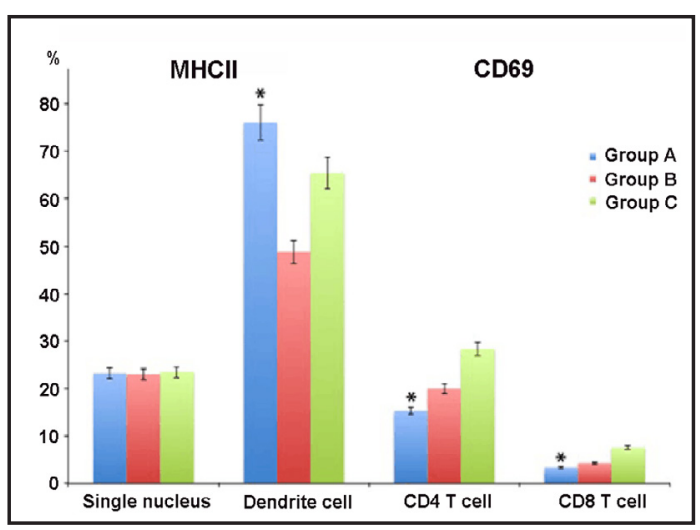

Fig. 4. MHCII and CD69 expression marker evaluation shows that the differences between the extracellular vesicle group and the other two groups were apparent, * represents statistical significance compared with groups A and B.

Fig. 5. The leukocyte infiltration in bilateral hemisphere of each group, with CD45 as the marker. There was no apparent significant difference in the ipsilateral cerebral hemispheres among the three groups.

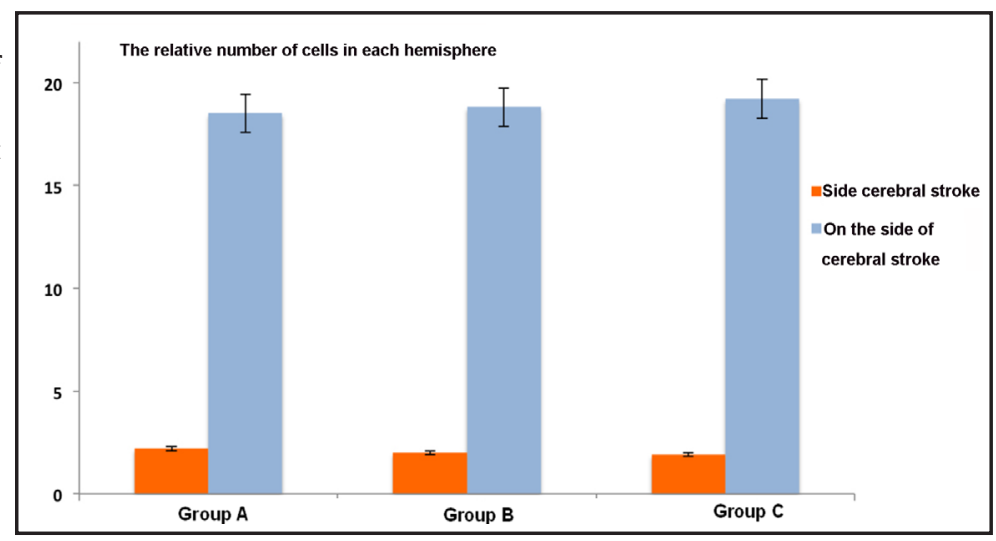

neuronal cells marked with mature or immature BrdU in these two groups was significantly increased. The quantity of $\mathrm{CD} 31+/ \mathrm{BrdU}+$ cell expression also increased, which indicated that new endothelial cells were produced; however, there was no significant difference between the MSC and extracellular vesicle groups (Fig. 3).

\section{Effect of extracellular vesicles on the immune response}

First, we detected the absolute cell number of leukocyte subsets in the blood. Because the systemic immune response induced by cerebral apoplexy was time-dependent, we mainly detected immunologic function 1 week after cerebral apoplexy. Compared with the control group, there was no significant difference in the quantity of B cells, NK cells, and T cells between groups A and B, whereas the quantity of hyaline leukocytes was significantly reduced. Although the number of dendritic cells did not change significantly between groups A and B, the expression of MHC II dendritic cells in the extracellular vesicle group was clearly reduced. In comparing the blank control and extracellular vesicle groups, the quantity of CD4+ and CD8+T cells was increased significantly, suggesting that extracellular vesicles reduced the composition and activated state of peripheral blood cells after focal cerebral ischemia (Table 1 and Fig. 4).

Effect of extracellular vesicles on immune cell infiltration of brain tissue

Inflammatory reaction of the brain can aggravate the severity of secondary brain injury after cerebral apoplexy, which is mainly characterized by different types of immune cells 


\section{Cellular Physiology Cell Physiol Biochem 2016;40:155-162 \\ \begin{tabular}{l|l} 
and Biochemistry Published online: November 18, 2016 & $\begin{array}{l}\text { (c) } 2016 \text { The Author(s). Published by S. Karger AG, Basel } \\
\text { www.karger.com/cpb }\end{array}$
\end{tabular} \\ Hu et al.: Extracellular Vesicles Facilitate Regeneration}

infiltrating into brain tissue. First, we analyzed CD45+ leukocytes by immunohistochemical staining, which excluded the potential confounding effects caused by infarct size differences. We found no significant differences in the extracellular vesicles at two time points. Because CD45 staining could not distinguish between different hematopoietic cell subsets, we processed multi-parameter flow cytometry for rat brain cells at 1 week. The level of CD45 expression in the peripheral blood leucocytes was significantly higher than microglia. The results of quantitative analysis showed that the quantity of leukocytes in the unilateral hemisphere was significantly higher than the contralateral hemisphere, which was consistent with the immunohistochemical findings. Extracellular vesicles exerted no significant effect on cerebral hemisphere infarction immersed by leukocytes (Fig. 5).

\section{Discussion}

In this study, we preliminarily showed that MSC and extracellular vesicle mitigate nervous dysfunction after cerebral ischemic stroke, which is probably correlated with promoting the regeneration of nerve and blood vessels as previously reported related to MSC transplantation. It has been reported that MSCs and vesicals are vital regulatory factors of immune function, and we found that the level of B cell, NK cell, and T leukomonocyte expression in group B was slightly decreased with no statistical significance when compared with groups $\mathrm{A}$ and $\mathrm{C}$.

MSCs have been confirmed to induce nerve protection. Many categories of cerebral apoplexy models have shown that nerve protection was primarily terminated through an indirect mechanism. Recent studies have reported that stem cell-derived extracellular vesicles exert an effect on alleviating movement coordination disorders after focal cerebral ischemia $[10,11]$. In the current study, we compared and found that extracellular vesicles and MSCs possess an identical effect. Extracellular vesicles and MSCs can increase the quantity of surviving neurons surrounding ischemic brain tissues. An early and terminal neuroprotective effect could increase neuron survival, so we analyzed the ischemia injury 1 week after infarction, but we did not find the effect of extracellular vesicles on the infarct area. A previous study confirms that MSCs can reduce infarction, which was closely related with the time points of cell transplantation. Thus, administering extracellular vesicles during early stroke do not prevent infarction; however, promoting neural rehabilitation and preventing secondary neurodegeneration might primarily affect the sub-acute stage after infarction. Intravenous injection of extracellular vesicles could induce a neuroprotective effect after infarction.

The degree to which MSCs and precursor cells integrate into the neural network signal is extremely limited, but the main function is to promote remodeling of brain tissue and regeneration of nerve and blood vessels through an indirect effect. According to our study, extracellular vesicles exert the same effect, but the specific mechanism is not clear. It has been reported that transplantation could improve the microenvironment after apoplexia and upregulate the level of related growth factors to promote neuranagenesis after apoplexia [1216]. Extracellular vesicles might have similar effects, depending on the content. The complex interaction between extracellular vesicles and the host plays a key role during this progress instead of single growth factors or a small molecule RNA effect. Still, identical mechanisms of extracellular vesicles and MSCs indicate that extracellular vesicles might be the main component of MSCs which play a role in clinical treatment. A recent study showed that the induction of angiogenesis is of importance for the neuroprotective effect of MSCs [17]. Thus, the improvement in nerve function, the neuroprotective effect, and revascularization were essentially the same.

MSCs possess immune inertia and regulate central and peripheral immune responses. MSCs exert a remodeling effect by immune reaction instead of combining with the structure directly into the brain. Analyses of kidney and myocardial injury models have demonstrated that injecting MSC-derived culture medium could produce an immunosuppressive action 
similar to MSCs [18-20]. Thus, we deduced that extracellular vesicles can also regulate central and peripheral immune responses. Immunologic suppression appears for a period of time with cerebral apoplexy, which increases the susceptibility of infection and neural degeneration. We deduced that anti-immune activity of extracellular vesicles could aggravate immunologic deficiency after apoplexia, which might worsen prognosis after apoplexy. Our study results showed that the extracellular vesicles were not aggravated, rather reduced lymphocytopenia after ischemia, which is the main indication of immunologic suppression after apoplexy. After transplantation of extracellular vesicles, the quantity of B cells, NK cells, and T cells was all increased. Recent studies have confirmed that MSC transplantation can restrain inflammatory reactions involving the brain [21,22]. In the current study, we did not find that changes in cerebral stroke could induce infiltration of immune cells after extracellular vesicle transplantation, which is in contrast to previous reports. It has been reported that lymphocytic infiltration of brain tissues is decreased after delivery of MSCs and production of pro-inflammatory cytokines and nitric oxide is constrained, suggesting that MSCs exert no effect on immune stimulation [23]. The extracellular vesicles used in the current study were derived from non-stimulated MSCs, thus the anti-inflammatory activity might be too low to organize the immersion of leukomonocytes.

There were two limitations to acknowledge. First, only NeuN, a marker for mature neurons, was investigated. In a corollary study, we will analyze alternative markers for young neurons to further validate the findings. Second, immunosuppression studies are in urgent need of investigation.

In conclusion, both MSCs and extracellular vesicles accelerate the recovery of neural function after cerebral apoplexy in a rat model. Weakening immune suppression function contributes to remodeling of brain tissue.

\section{Acknowledgments}

This work was supported by grants from the Medical and health science and technology plan of Zhejiang Province (Funding N0.2013RCA036).

\section{Disclosure Statement}

All authors declare no conflict of interests.

\section{References}

1 Bacigaluppi M, Pluchino S, Peruzzotti-Jametti L, Kilic E, Kilic U, Salani G, Brambilla E, West MJ, Comi G, Martino G, Hermann DM: Delayed post-ischaemic neuroprotection following systemic neural stem cell transplantation involves multiple mechanisms. Brain 2009;132:2239-2251.

2 Banerjee S, Williamson DA, Habib N, Chataway J: The potential benefit of stem cell therapy after stroke: an update. Vasc Health Risk Manag 2012;8:569-580.

3 Savitz S I, Misra V, Kasam M, Juneja H, Cox CS Jr, Alderman S, Aisiku I, Kar S, Gee A, Grotta JC: Intravenous autologous bone marrow mononuclear cells for ischemic stroke. Ann Neurol 2011;70:59-69.

4 Jia R, Li J, Rui C, Ji H, Ding H, Lu Y, De W, Sun L: Comparative Proteomic Profile of the Human Umbilical Cord Blood Exosomes between Normal and Preeclampsia Pregnancies with High-Resolution Mass Spectrometry. Cell Physiol Biochem 2015;36:2299-2306.

5 Zhang J, Zhang HD, Yao YF, Zhong SL, Zhao JH, Tang JH: $\beta$-Elemene Reverses Chemoresistance of Breast Cancer Cells by Reducing Resistance Transmission via Exosomes. Cell Physiol Biochem 2015;36:22742286. 


\section{Cellular Physiology Cell Physiol Biochem 2016;40:155-162 \begin{tabular}{l|l|l} 
DOI: 10.1159/000452533 & $\begin{array}{l}\text { (c) 2016 The Author(s). Published by S. Karger AG, Basel } \\
\text { www.karger.com/cpb }\end{array}$
\end{tabular} \\ Hu et al.: Extracellular Vesicles Facilitate Regeneration}

6 Li H, Han L, Yang Z, Huang W, Zhang X, Gu Y, Li Y, Liu X, Zhou L, Hu J, Yu M, Yang J, Li Y, Zheng Y, Guo J, Han J, Li L: Differential Proteomic Analysis of Syncytiotrophoblast Extracellular Vesicles from Early-Onset Severe Preeclampsia, using 8-Plex iTRAQ Labeling Coupled with 2D Nano LC-MS/MS. Cell Physiol Biochem 2015;36:1116-1130.

7 Tsai WH, Shih CH, Feng SY, Li IT, Chang SC, Lin YC, Hsu HC: CX3CL1(+) microparticles mediate the chemoattraction of alveolar macrophages toward apoptotic acute promyelocytic leukemic cells. Cell Physiol Biochem 2014;33:594-604.

8 Ludwig A K, Giebel B: Exosomes: small vesicles participating in intercellular communication. Int J Biochem Cell Biol 2012;44:11-15.

9 Chen J, Li Y, Wang L, Zhang Z, Lu D, Lu M, Chopp M: Therapeutic benefit of intravenous administration of bone marrow stromal cells after cerebral ischemia in rats. Stroke 2001;32:1005-1011.

10 Dharmasaroja P: Bone marrow-derived mesenchymal stem cells for the treatment of ischemic stroke. J Clin Neurosci 2009;16:12-20.

11 Leong W K, Henshall TL, Arthur A, Kremer KL, Lewis MD, Helps SC, Field J, Hamilton-Bruce MA, Warming S, Manavis J, Vink R, Gronthos S, Koblar SA: Human adult dental pulp stem cells enhance poststroke functional recovery through non-neural replacement mechanisms. Stem Cells Transl Med 2012;1:177-187.

12 Schwarting S, Litwak S, Hao W, Bähr M, Weise J, Neumann H: Hematopoietic stem cells reduce postischemic inflammation and ameliorate ischemic brain injury. Stroke 2008;39:2867-2875.

13 Shen L H, Li Y, Chen J, Zhang J, Vanguri P, Borneman J, Chopp M: Intracarotid transplantation of bone marrow stromal cells increases axon-myelin remodeling after stroke. Neuroscience 2006;137:393-399.

14 Ukai R, Honmou O, Harada K, Houkin K, Hamada H, Kocsis JD: Mesenchymal stem cells derived from peripheral blood protects against ischemia. J Neurotrauma 2007;24:508-520.

15 Zheng W, Honmou O, Miyata K, Harada K, Suzuki J, Liu H, Houkin K, Hamada H, Kocsis JD: Therapeutic benefits of human mesenchymal stem cells derived from bone marrow after global cerebral ischemia. Brain Res 2010;1310:8-16.

16 England T, Martin P, Bath PM: Stem cells for enhancing recovery after stroke: a review. Int J Stroke 2009;4:101-110.

17 Yoo SW, Kim SS, Lee SY, Lee HS, Kim HS, Lee YD, Suh-Kim H: Mesenchymal stem cells promote proliferation of endogenous neural stem cells and survival of newborn cells in a rat stroke model. Exp Mol Med 2008;40:387-397.

18 Doeppner TR, Ewert TA, Tönges L, Herz J, Zechariah A, ElAli A, Ludwig AK, Giebel B, Nagel F, Dietz GP, Weise J, Hermann DM, Bähr M: Transduction of neural precursor cells with TAT-heat shock protein 70 chaperone:therapeutic potential against ischemic stroke after intrastriatal and systemic transplantation. Stem Cells 2012;30:1297-1310.

19 Timmers L, Lim SK, Arslan F, Armstrong JS, Hoefer IE, Doevendans PA, Piek JJ, El Oakley RM, Choo A, Lee CN, Pasterkamp G, de Kleijn DP: Reduction of myocardial infarct size by human mesenchymal stem cell conditioned medium. Stem Cell Res 2007;1:129-137.

20 Hsieh JY, Wang HW, Chang SJ, Liao KH, Lee IH, Lin WS, Wu CH, Lin WY, Cheng SM: Mesenchymal stem cells from human umbilical cord express preferentially secretedfactors related to neuroprotection, neurogenesis, and angiogenesis. PLoS One 2013;8:e72604.

21 Scheibe F, Klein 0, Klose J, Priller J: Mesenchymal stromal cells rescue cortical neurons from apoptotic cell death in an in vitro model of cerebral ischemia. Cell Mol Neurobiol 2012;32:567-576.

22 Tate CC, Fonck C, McGrogan M, Case CC: Human mesenchymal stromal cells and their derivative, SB623 cells, rescue neural cells via trophic support following in vitro ischemia. Cell Transplant 2010;19:973-984.

23 Cantaluppi V, Gatti S, Medica D, Figliolini F, Bruno S, Deregibus MC, Sordi A, Biancone L, Tetta C, Camussi G: Microvesicles derived from endothelial progenitor cells protect the kidney from ischemia-reperfusion injury by microRNA-dependent reprogramming of resident renal cells. Kidney Int 2012;82:412-427. 